\title{
Gamificación en la educación: Experiencia basada en la diversidad ecuatoriana.
}

Gamification in education: Experience based on Ecuadorian diversity.

Carlos Luis Sánchez Pacheco

Fecha de recepción: 06 de noviembre 2020

Fecha de aceptación: 21 de diciembre 2020 


\title{
Gamificación en la educación: Experiencia basada en la diversidad ecuatoriana.
}

\author{
Gamification in education: Experience based on Ecuadorian diversity.
}

Carlos Luis Sánchez Pacheco ${ }^{1}$.

Como citar: Sánchez, C. (2021). Gamificación en la educación: Experiencia basada en la diversidad ecuatoriana Revista Universidad de Guayaquil. 132(1). 21-32. DOI:

https://doi.org/10.53591/rug.v132i1.1357

\section{RESUMEN}

Este presente artículo aborda la Gamificación como práctica educativa a manera de experiencia en la diversidad ecuatoriana. Comenzamos por delinear las características de lo que hoy se describe como Cultura Digital y sus implicaciones para el entorno escolar; luego analizamos la consecuente necesidad de un cambio en las prácticas pedagógicas. Luego, como alternativa a este cambio, proponemos la Gamificación escolar, una estrategia que consiste en insertar elementos y estética de los juegos electrónicos en el aula para trabajar los contenidos de una manera más amena la cual motiva al jugador a descubrir nuevas formas de permanecer en la educación. Dada la gran cantidad de personas de todas las edades que se encuentran inmersas en el mundo de los juegos y los expresivos resultados que ya ha obtenido el ámbito corporativo, especialmente el Marketing, con prácticas gamificadas, se cree que esta estrategia puede contribuir a un aprendizaje efectivo dada su potencia para involucrar a las personas a través de tareas placenteras, desafiantes e intrigantes, bajo la etiqueta de misiones. El artículo finaliza con la afirmación de que, para implementar la gamificación en las prácticas pedagógicas, los docentes necesitan conocer y comprender la lógica de los juegos electrónicos y su funcionamiento. Solo así podrán reproducir la mecánica de estos juegos en el aula de forma analógica.

Palabras clave: gamificación; contenido escolar; aprendizaje; diversidad; educación; juegos serios

\section{ABSTRACT}

This present article addresses Gamification as an educational practice as an experience in Ecuadorian diversity. We begin by outlining the characteristics of what is now described as Digital Culture and its implications for the school environment; then we analyze the consequent need for a change in pedagogical practices. Then, as an alternative to this change, we propose School Gamification, a strategy that consists of inserting elements and aesthetics of electronic games in the classroom to work the contents in a more enjoyable way, which motivates the player to discover new ways of staying in the education. Given the large number of people of all ages who are immersed in the world of games and the expressive results that the corporate sphere has already obtained, especially Marketing, with gamified practices, it is believed that this strategy can contribute to learning effective given its power to involve people through pleasant, challenging and intriguing tasks, under the label of missions. The article ends with the statement that, to implement gamification in pedagogical practices, teachers need to know and understand the logic of electronic games and how they work. Only then will they be able to reproduce the mechanics of these games in the classroom in an analog way.

Keywords: gamification; school content; learning; diversity; education; serious games

${ }^{1}$ Doctor y Magister, Sociedad de Investigación Pedagógica Innovar, Colombia, Correo electrónico: carlossanchez21@hotmail.com, ORCID: https://orcid.org/0000-0003-4831-5813 


\section{INTRODUCCIÓN}

Esto significa que las acciones y reacciones humanas han ido cambiando debido a la interacción con los diversos dispositivos, gadgets, herramientas y software que se están creando en los últimos años. Según (Harvey, 1990), en los últimos años hemos estado viviendo una "fase intensa de comprensión del espacio-tiempo que ha tenido un impacto desorientado y disruptivo (...) en la vida social y cultural", es decir, vivimos en un mundo caracterizado por la aceleración de los cambios, desde el advenimiento de lo que podemos llamar Cultura Digital.

Lo que vemos, en realidad, es que, si bien las empresas de marketing ya se han dado cuenta de las transformaciones sociales que estamos viviendo, en este marcado movimiento online, y están explorando todo el potencial de los medios digitales para vender y promocionar sus productos, la escuela aún permanece en su secuencialidad física, estructural y formal, favoreciendo, en la mayoría de los casos, una lectura recortada de fragmentos sellados en libros impresos, basada en clases secuenciales y cerradas, bajo estructuras curriculares lineales (Martins, 2013). En verdad, es necesario darse cuenta de que mientras el mundo exterior a la escuela está plagado de información, ruido y emoción, todavía hoy se espera una escuela con aulas silenciosas, silenciosas, con escritorios alineados, preferiblemente sin comunicación entre los estudiantes durante el horario escolar. (Martins, 2013)

Sin embargo, esta estructura se está volviendo insostenible. Los profesores no se contentan con el descuido y la falta de preparación de sus alumnos; los niños y jóvenes ya no pueden soportar pasar horas y horas atrapadas en un espacio limitado con una lluvia de información desconectada de su realidad y sin un significado que les agregue valor. Esta escuela, por supuesto, está en contra de su voluntad, creando descontento entre los jóvenes y los niños, que ya no comprenden su importancia en su educación.

En este sentido, es urgente no solo reevaluar el currículo básico que se formuló para la escuela en su estructura industrial cartesiana, sino también repensar las prácticas de enseñanza metodológica. Es este último punto el que se discutirá en este artículo.

\section{¿Juegos en la escuela?}

De los elementos que caracterizan la cultura digital, los juegos electrónicos tienen una profunda influencia en la vida de un número considerable de personas, especialmente las que nacieron en este mundo tecnológico digital. Los juegos pueden, en su mayor parte, ser considerados hoy como experiencias inmersivas como el cine y la literatura con formatos e historias cada vez más complejos. Quizás, por tanto, las cifras sean impresionantes.

Según (García, 2019), los videojuegos representan hoy el mercado de entretenimiento más grande del mundo, superando a la industria del cine y la música en conjunto. Según (Fardo, 2013), en Estados Unidos prácticamente todos los hogares cuentan con un dispositivo capaz de ejecutar juegos comerciales, la mitad de las casas tienen un dispositivo dedicado solo a juegos y la edad promedio de los jugadores es de 30 años. En Ecuador, según la encuesta de la UCSG del 2018, el 42\% de las mujeres y el 58\% de los hombres declaran jugar un juego, en multiplataformas.

Ante esta realidad indiscutible que muestran los números, no podemos, como educadores, negar la importancia de los juegos en la vida de nuestros alumnos y, en consecuencia, su importancia en la educación. Tal importancia no se limita al hecho de que mucha gente juega, sino principalmente al aporte cognitivo que estos confieren a los jugadores. Ya existen varios estudios que demuestran que los juegos tienen potencial educativo. Se ha comprobado que los jugadores pueden mejorar en 
razonamiento lógico, observación, espacialidad, resolución de problemas, lectura, toma de decisiones (Prensky, 2006; Burke, 2015). Aquí en Ecuador, los estudios ya han demostrado los beneficios de, por ejemplo, aprender inglés (Sánchez-Pacheco C. L., 2019).

Según (Alves, 2015; Deterding, 2012; Dichev \& Dicheva, 2017), en los juegos, la interacción que establecen los jugadores "permite a los jóvenes tener habilidades fundamentales para el éxito del proceso de enseñanza y aprendizaje en la medida en que les brindan habilidades y competencias para mantenerse 'vivos' en la vida y en el mundo del trabajo". Para (Gee, 2009), en sus estudios sobre juegos, afirma que los seres humanos que nunca enfrentan desafíos y frustraciones, que nunca desarrollan nuevos estilos de aprendizaje y que nunca enfrentan situaciones de fracaso directamente pueden, al final, volverse más pobres, gente más limitada. Para el autor, la cultura digital de los juegos lleva a las personas a creer que dichos entornos establecen una lógica diferenciada, ya sea a través del entretenimiento o como la posibilidad de constituir espacios de aprendizaje para diferentes tipos de conocimiento (afectivo, cognitivo, social, cultural, entre otros).

Todo lo que se ha expuesto hasta ahora explica la importancia de utilizar juegos en la educación. Sin embargo, no se propone aquí la transformación de la escuela en casas especializadas en juegos, ni la dependencia de dispositivos electrónicos y tecnología digital, sino la inserción de la mecánica o estética de los juegos (gamificación) en el aula, con el fin de enseñar contenidos, desarrollar un sentido crítico, ayudar en la toma de decisiones, desarrollar un sentido de cooperación y colaboración, creatividad, entre otras habilidades que conforman lo que comúnmente se entiende como aprendizaje significativo. Uno de los aspectos más destacados es la forma placentera, que favorece el compromiso en la búsqueda del conocimiento y el desarrollo del sentido de pertenencia a la escuela.

\section{¿Qué es la gamificación?}

Existen algunos desacuerdos sobre el origen del término gamificación. Según el autor, Nick Pelling, programador informático e investigador británico, fue quien acuñó este neologismo por primera vez en 2002. Sin embargo, fue con Jane McGonigal que en 2010, el término se hizo popular (SánchezPacheco C. , 2020).

Del inglés, gamificación, encontramos, en nuestra lengua materna, tres variantes: gamificación (la más utilizada), gamificación y ludificación. Adoptaremos el término más común con la definición de (Fardo, 2013; Kapp, 2012), en una de las primeras encuestas en Educación sobre el tema. Según el autor, en definitiva, la gamificación es un fenómeno que consiste en "el uso de elementos, estrategias y pensamientos de juegos fuera del contexto de un juego, con el fin de contribuir a la resolución de algún problema". Esta definición no difiere mucho de las encontradas por autores en el campo del diseño de juegos o empresas. Está claro que el mayor deseo es intentar involucrar a las personas en un mismo objetivo, a través de una competencia sana que genere cada vez más motivación.

Algunos casos ya están destacando en el mundo empresarial, como el de Volkswagen, que instaló dispositivos de sonido y visuales en los escalones de una escalera del metro en Estocolmo, Suecia, simulando un piano. La gente empezó a subir y bajar divirtiéndose, lo que generó el popular apodo de "teoría de la diversión". Esta novedad propició un cambio en la forma de pensar y actuar de los transeúntes. La gente empezó a hacer más ejercicio debido al juego (Yee, 2007). Eligieron la escalera musical en lugar de la comodidad de la escalera mecánica. Otro ejemplo comercial es el de $M c$ Donald's, que con sus promociones y obsequios infantiles favorece al consumidor, que se apega al deseo de completar las colecciones de juguetes.

Quizás una de las aplicaciones para educación más interesantes, hasta ahora, es Duolingo, que se puede usar en sistemas Android o IOS, para aprender idiomas extranjeros. El inglés se aprende del español, pero de este idioma puedes aprender una variedad de otros, como portugués, francés, alemán, italiano, etc. 
Aunque se ha destacado ejemplos que se basan en el uso de dispositivos electrónicos, la gamificación de una actividad no presupone el uso de tecnologías digitales. Puede ocurrir, por ejemplo, en espacios escolares sin tales recursos disponibles. Simplemente organice las actividades según los criterios fundamentales de un juego, entre ellos, las reglas, la retroalimentación rápida, el desafío, la competencia y la recompensa, apuntando a los objetivos pedagógicos a alcanzar (Martínez, 2017).

\section{¿Por qué gamificar el contenido escolar?}

El juego es agradable y cautivador. Implica quién está jugando en un "círculo mágico" (Huizinga, 2010), motiva al jugador a descubrir nuevas formas de permanecer en ese círculo.

Basado en varios autores, (Zichermann \& Cunningham, 2011; Rodríguez \& Santiago, 2015) describen el juego como un sistema en el que los jugadores se involucran en un conflicto artificial, con reglas, y que genera un resultado cuantificable. Para (Fardo, 2013), el aula puede equipararse a un juego. Si comparamos el aula con la definición de (Zichermann \& Cunningham, 2011), veremos que realmente existe un conflicto artificial para la construcción del aprendizaje, con reglas de conducta, producción de contenidos, etc. y resultado cuantificable. El problema es que, la mayoría de las veces, el niño que está en esa aula no está ahí por voluntad o interés propio, sintiéndose motivado, necesidad básica de sumergirse, de hecho, en el "círculo mágico".

Johann Huizinga, precursor de sus análisis y uno de los más grandes investigadores de juegos del siglo XX, afirma que el juego es anterior a la cultura y a su creador. Para este filósofo holandés, "el juego es una función de la vida" (Huizinga, 2010). A partir de este análisis, y dándose cuenta desde hace años, en las prácticas docentes, de cuánto niños y jóvenes están inmersos en su vida cotidiana en un mundo lúdico de redes sociales, videos y juegos, y también descubriendo, a través de la convivencia informal con varios niños, principalmente requiere y parece promover el desarrollo de habilidades consideradas importantes para la sociedad del siglo XXI, se cree que es de gran importancia revisar las prácticas de aula con respecto no solo a los objetos de aprendizaje que se utilizan, así como a los prácticas metodológicas que se construyen (Zúñiga, 2019). En otras palabras, no solo se necesita traer juegos, dispositivos, software y aplicaciones que son parte del día a día de los jóvenes al aula para recrear el proceso de enseñanza-aprendizaje, sino también replantear prácticas en base a interacciones que están funcionando y principalmente, están despertando el interés y fomentando el aprendizaje en quienes los utilizan.

Tomando, entonces, la idea desarrollada por Roger Caillois, lector y crítico de Huizinga, en el libro El juego y el hombre, de que el juego aumenta la capacidad del individuo para superar obstáculos, dificultades y lo introduce en la vida; y recuperar la importancia que Huizinga le da al juego en la sociedad y también en la edad adulta, es que la gamificación se nos aparece como una nueva estrategia de enseñanza. Después de todo, si desde Huizinga varios autores han coincidido en que todo juego significa algo, es necesario investigar qué significan los elementos de los juegos para los jóvenes de hoy y hacer uso de ellos, a favor de la educación.

Por eso partiendo del argumento piagetiano de que la comprensión presupone creación, es decir, el aprendizaje debe partir siempre de una acción real y material (Iquise \& Rivera, 2020). Según esta concepción, el desarrollo del niño es un proceso continuo, subordinado a la acción del sujeto y su interacción con los objetos. De ahí la validez y el beneficio de las actividades gamificadas.

La experiencia del profesor de Design, Lee Sheldon, a partir del análisis del libro "El aula multijugador: diseñando cursos como un juego", realizado por (Fardo, 2013; Zepeda, Abascal, \& López, 2016), como parte de su investigación sobre gamificación. En él, Fardo informa que Sheldon aplicó los conceptos del juego MMORPG en su aula, creando personajes, grupos, misiones, sistemas de puntuación, formularios de retroalimentación y misiones en solitario, para darle al estudiante la libertad de trabajar en equipo o individualmente, dejándote sentir libre de aprender. 
Se tiene que ver que, mucho más que cambiar los materiales o espacios en el aula o en la escuela, es necesario repensar la forma en que estamos tratando los contenidos, que quizás, desde hace mucho tiempo, están sobrevalorados a expensas del aprendizaje. Y eso recae sobre uno de los pilares de la educación como lo es el docente (Mite, 2020). Desarrollar prácticas gamificadas requiere, por parte del docente, el conocimiento de la estructura y mecánica de los juegos, además de tener una actitud proactiva de creación y recreación de actividades variadas y en número suficiente para dar cuenta de la gran interacción que el juego proporciona (Vera, 2020). Pero hay que tener cuidado de no utilizar la nueva estrategia como "el aspecto principal de la experiencia de aprendizaje de los estudiantes" (Massarolo \& Mesquita, 2013; Oliva, 2016) y caer prácticamente en el mismo error que ya hemos vivido, resaltando estrategia o contenido en lugar de aprendizaje.

\section{Los elementos básicos de un juego}

Ya se han realizado muchos estudios e investigaciones para identificar qué mecanismos y características de los juegos digitales cautivan a los seres humanos, especialmente a los jóvenes, (McGonigal, 2012) destaca cuatro características: objetivo, reglas, sistema de retroalimentación y participación voluntaria. En particular, quisiera destacar este último, ya que, sin el acto de querer, puede haber, como máximo, un pseudoaprendizaje para realizar una determinada actividad, pero que, en poco tiempo, deja de existir. Y esto es lo que más vemos en la escuela.

Para (Werbach \& Hunter, 2012) la dinámica (restricciones, emociones, narrativa, progresión y relaciones) en los juegos, mecánicas (desafíos, oportunidades, competencia, cooperación, comentarios, adquisición de recursos, recompensas, negociaciones entre jugadores, ciclos o rondas del juego y estado de ganador) y los componentes (logros, avatares, insignias, choque, desbloqueo de contenido, niveles, misiones, gráficos sociales y equipos). Y existe una relación entre ellos. Cada mecánica está vinculada a una o más dinámicas, y cada componente está vinculado a una o más mecánicas o dinámicas.

Para comprender un poco sobre gamificación, es necesario comprender los elementos de los juegos, que dan lugar a la actividad gamificada. La tabla 1 resume las principales características de los juegos.

\section{Por una propuesta de gamificación como estrategia de enseñanza-aprendizaje}

El análisis cuidadoso de cada uno de estos elementos proporciona información valiosa que apoyará el trabajo del docente a la hora de desarrollar una práctica gamificada. Así, a la hora de planificar una actividad de esta naturaleza, el docente debe ser consciente de todos los elementos que pueden favorecer o dificultar la ejecución del juego, sabiendo cómo cada uno de ellos afecta a la actividad. Preguntas como "¿Cómo afecta la combinación de una estructura de juego y un escenario en particular al juego?" siempre deben orientar las decisiones del profesor al planificar sus actividades. Aun así, las preguntas sobre las consecuencias que ciertas reglas pueden traer al juego son igualmente importantes (Rodríguez \& Santiago, 2015). En algunas situaciones, una cierta regla puede limitar tanto las acciones de los jugadores, que su evolución puede verse seriamente afectada; en otros, la no delimitación de una regla puede convertir el juego en una actividad tan libre que no generará una buena jugabilidad.

Dado su carácter lúdico, el juego debe contener un contenido de ficción atractivo, inteligente y coherente con el propósito para el que se propone. Así, la narrativa adquiere una gran importancia en una práctica gamificada. También debe ser coherente con el escenario en el que se producirán batallas o disputas. Por tanto, a la hora de planificar una práctica gamificada, el docente debe prestar atención a la narrativa que, en el caso de una práctica escolar cuyo objetivo es la adquisición de ciertos conocimientos, debe incluir lúdico, concepción topológica del tema a aprender y todo ello. sin perder de vista el rigor del conocimiento científico de la disciplina (Ortiz, Jordán, \& Agredal, 2018). La narrativa tiene el poder de involucrar al jugador en un esfuerzo por desvelar la historia del héroe 
recorriendo sus caminos y experimentando sus amores y problemas con él, y es así, viviendo la vida de su héroe o avatar, que el jugador se sumerge en un universo paralelo asimilando sus aventuras, historias, amigos y enemigos, en definitiva, el contenido a aprender cuando se trata de una práctica pedagógica ludificada (Kim \& Lee, 2015).

Además de los aspectos planteados anteriormente, hay otros elementos que deben estar igualmente equilibrados para garantizar la jugabilidad. Un juego no siempre tiene una secuencia lineal de dificultad. Al contrario. Para ser intrigante, emocionante y placentero, el juego debe escalar en niveles de desafíos, comenzando desde el más simple hasta el más complejo, desde el más fácil hasta el más difícil, y así se construirá la progresión del jugador, que ahora puede estar en niveles bien evolucionados ahora, debido a un error estratégico, falta de habilidad o conocimiento en el cumplimiento de una determinada misión, puede retroceder o ser superado por su oponente. Esta movilidad de niveles garantiza, junto con los demás elementos, el placer de jugar y ganar desafíos. También es importante destacar que todo juego debe respetar el principio de equidad y, mientras tanto, la retroalimentación, la retroalimentación que se da a la acción de un jugador, necesita, además de estar presente, ser clara, sin dejar lugar a dudas. Debe ser justo e inmediato. De esta forma, se garantiza la transparencia del juego y el cumplimiento de las reglas propuestas.

En una acción pedagógica ludificada, el profesor debe asegurarse de que el sistema de retroalimentación sea claro para todos los estudiantes-jugadores (Sánchez \& Colomer, 2018). Muy cerca de la retroalimentación en cuanto a suavidad y transparencia, la puntuación del jugador debe ser visible para todos los demás participantes y debe servir, además de transparencia, como elemento motivador para lograr los objetivos, ya sea para adelantarse a otros oponentes, bien para lograrlos, o para superar sus propias limitaciones y dificultades en un proceso de constante motivación. Todo jugador juega siempre por placer de ganar la recompensa, aunque sea un simple emblema que lograste pasar por otra etapa. Después de todo, el placer de jugar, competir individualmente o en grupos, a menudo en actitud de cooperación, duelo, reír, emocionarse, estresarse, estar en busca de lo que representará la victoria, la conquista sobre los oponentes, la recompensa. Es por eso que debería estar detallado al principio (Putz, Hofbauer, \& Treiblmaier, 2020). Debe quedar claro para el estudiante jugador lo que obtendrá al participar en un viaje lleno de misiones, desafíos, niveles de dificultad, reglas y puntajes (Meşe \& Dursun, 2018). En el caso de una actividad en el aula, el profesor también debe dejar claro qué recompensa obtendrá el ganador o grupo de ganadores por su esfuerzo. Sin esto, la competencia y la colaboración entre compañeros no tienen sentido y la consumación del placer de jugar puede frustrarse.

Gamificar, entonces, una actividad pedagógica presupone el cumplimiento de unos requisitos. Además de poder actuar desde los elementos básicos de los juegos, (Simões, Redondo, \& Fernández Vilas, 2013) también sugieren seguir algunos pasos que destacamos aquí:

- Pensar y proyectar la actividad, es decir, "jugar" en papel todo lo que pueda describir la estrategia y luego buscar ideas sobre cómo utilizar los elementos del juego en ella.

- Trabaje siempre con la posibilidad de experimentación. Como en el juego, en el que el jugador aprende mucho en la relación ensayo-error, en una actividad gamificada es interesante tener esa libertad.

- Cree una forma de dar retroalimentación rápida y eficiente, rompiendo la idea de tener una devolución al cabo de dos meses. Esto no solo puede ser demasiado tarde para corregir errores, sino que a menudo también es muy desalentador.

- Adapte las tareas al nivel de habilidad de los estudiantes o grupos de estudiantes, por ejemplo: subdividir las tareas difíciles en varias más pequeñas o permitir diferentes caminos hacia el éxito.

- Poner la diversión y el placer como parte integral de la actividad (pero con la conciencia de que el interés y significado de la actividad para el alumno es lo que promueve la motivación 
real) para que sea algo más motivador para el aprendizaje. Puedes cambiar un poco el sistema de recompensas por esto.

- Es un gran desafío poner todo esto en práctica, pero como el docente es capaz de percibir en la gamificación una posibilidad de enriquecer y hacer más efectiva la educación tanto para el alumno como para el educador, no existirán barreras para el uso de esta nueva estrategia de enseñanza - aprendizaje.

\section{MATERIALES Y MÉTODOS}

Frente a la globalización que vivimos y los medios de comunicación que venden una imagen ideal de belleza en la que todos deberían verse muy parecidos la enseñanza de la cultura ecuatoriana, basada en las diversidades étnicas y regionales hace obligatoria la enseñanza intercultural para no perder el vínculo con nuestros orígenes. Principalmente porque todo lo que viene de fuera, incluso por la repercusión que se produce en las redes sociales, ha parecido cada vez más interesante.

Entonces, pensando en estos temas referentes a la interculturalidad ecuatoriana, se observó en la gamificación la posibilidad de no solo potenciar el aprendizaje de este contenido, así como desarrollar en los niños el gusto y el interés por estos temas, para no volverse más contenido que pasa y luego se olvida (Álvarez, 2020; Carrión, 2018). De esta manera, creamos y experimentamos lo siguiente:

En una estructura de competencia, a fines de octubre, período en el que muchos comenzaban a hablar sobre Halloween, la clase se dividió en cuatro equipos que pronto fueron introducidos a una narrativa que desafió a los participantes a recordar el Día de la Interculturalidad (12 de octubre). Así, como parte de la propuesta de actividad, insertada en esta construcción narrativa, se lanzó la primera misión de los equipos: buscar los códigos (imágenes) que estaban esparcidos por la sala y en los espacios más allá del aula para descifrar un texto que les diera un mapa para conquistar el territorio de nuestra cultura (Contreras \& Eguia, 2017; Cuenca \& Jiménez, 2018).

\section{Desarrollo}

Esta primera misión fue muy interesante porque se preveía una competencia entre los equipos, sin posibilidad de colaboración entre ellos, al fin y al cabo, quien descifrara el texto primero ganaría la primera etapa, dominando las regiones del país, así que nos dimos cuenta de que funcionaba el marcador motivando también el conocimiento y el interés por los mapas (Contreras-Espinosa, 2016). Sin embargo, los equipos utilizaron los códigos de los otros equipos que encontraron para hacer una especie de trueque de información, es decir, si un equipo conocía el código del otro, lo transmitía a cambio de su propio código. Se produjo, por tanto, una recreación por parte de los alumnos actores de la actividad en un proceso de mutua colaboración. Y todos habían entrado en el "círculo mágico" del juego.

En posesión de los territorios, según la puntuación obtenida por cada equipo (se estaban explicando las reglas de puntuación), se les presentó una nueva misión, continuando la narrativa creada. Los equipos deben recoger nuevos códigos en una mesa para desvelarlos, presentando, a través de un dibujo realizado en el acto, un avatar que representa a los seres legendarios a los que se refieren las descripciones (Gatti, Ulrich, \& Seele, 2019). Para aclarar a qué seres se refería dicha información, pudieron buscar en libros y en internet a través de sus dispositivos electrónicos.

Si en la primera misión el objetivo, además de conocer el contenido del texto descifrado, era insertar a los miembros del equipo en el círculo mágico, ganándolos así para la actividad propuesta, en esta teníamos como meta transformar los participantes en autores. La puntuación, en esta etapa, se otorgó según dos criterios: la rapidez con la que descifraron los rompecabezas y entregaron los avatares listos y la elección (votos de todos los participantes) del diseño que mejor caracterizó al legendario ser descrito.

Después de ocupar las cuatro regiones del Ecuador, los equipos fueron enviados a través de la 
continuación de la narrativa a una nueva etapa. Antes, sin embargo, cada equipo tenía que elegir uno de sus diseños para nombrar su grupo y caracterizar el "peón" del juego de mesa humano que comenzaría a continuación. En un recorrido por el conocimiento de nuestra cultura, cada equipo, después de sortear tirando los dados para decidir el orden de los jugadores, fue conducido a avanzar su "peón", desde el empezar a la línea de meta. Para moverse, los peatones lanzaron un dado grande al piso que informaba el número de casas que debían caminar si respondían correctamente a la pregunta que aparecía en el data-show al azar.

Fue interesante notar que la elección de las regiones del Ecuador generó la expectativa de una estrategia fronteriza, que sería bastante interesante para la continuación de la actividad en otros momentos. El uso de esta forma de puntuación nos mostró que lo más importante para ellos no era la victoria por la victoria, sino la posibilidad de crear estrategias y desarrollar un aprendizaje significativo.

\section{RESULTADOS Y DISCUSIÓN}

La investigación muestra los siguientes hallazgos de relevancia para el Talento Humano en aras de Si bien la actividad fue diseñada para alumnos del nivel de Básica Media (5to., 6to. y 7mo. grado), se puso a prueba en un curso del nivel de Bachillerato. De hecho, es una actividad que permite pasear por los diferentes grupos de edad, solo necesitando dirigir las preguntas del juego de mesa y los puzzles de las misiones uno y dos a un nivel más difícil o medio a fácil.

Esta experimentación fue muy útil porque el grupo de estudiantes - jugadores, aunque adultos y de diferentes áreas, realmente se involucró en la actividad, confirmando su compromiso al ingresar a "magia circulo ". Así quedó claro en el testimonio de un alumno de la clase que, al finalizar la actividad, participó y colaboró con un análisis crítico de la estrategia. Según ella, la actividad gamificada resultó muy interesante y adecuada para el año al que estaba destinada, también con la flexibilidad de edades como aspecto positivo. Además, agregó que la temática fue muy relevante "Me parece genial, por el contenido que vale la pena canjear en las unidades escolares" y luego dijo: "Me gustó mucho la parte que trae el personaje al juego y de esa manera el contenido se está dando de forma lúdica". El alumno también destacó el interés generado: "todo el tiempo movilizó a la clase, todos se involucraron" y también demostró los desafíos a superar: "la cuestión del dibujo fue un desafío, porque en nuestro grupo no había nadie con habilidad para dibujar, pero queríamos hacer lo mejor".

Lo que percibimos, en la práctica, durante la creación de la actividad y su aplicación es que una actividad gamificada en la escuela puede ser una estrategia para trabajar, de hecho, la transdisciplinariedad. En esta propuesta para el desarrollo de contenidos referentes a la interculturalidad ecuatoriana. En el puntaje propuesto, cada punto ganado representaba una pieza para que los equipos ocuparan las regiones. Una vez finalizado un equipo podría empezar a compartir el mismo espacio con otro (s), lo que resultaría en una relación fraccional, contenido matemático del sexto grado y revisión del séptimo.

También se recibió otras retroalimentaciones al final de la actividad gamificada realizada. Los jugadores elogiaron los desafíos bien definidos; claridad en la presentación de los objetivos a alcanzar; la narrativa bien construida, que lleva a los estudiantes a ingresar a la actividad como actores; retroalimentación con respuestas inmediatas a preguntas que no pudieron resolver; la presencia de contenidos curriculares aprendidos de la alegría; la transdisciplinariedad del juego, que abordó aspectos de Geografía, Historia, Matemáticas, entre otros; la variedad de habilidades requeridas y también desarrolladas; el dinamismo de la actividad, con posibilidad de superar el espacio restringido del aula; fomentar la cooperación entre los jugadores del grupo y la colaboración entre los grupos; presencia de niveles de dificultad, con progresión. Como aspectos que es necesario mejorar, los jugadores destacaron que el sistema de puntuación no estaba claro. Del mismo modo, las recompensas podrían resolverse mejor. 


\section{CONCLUSIONES}

En este artículo se ha visto que los avances que se han producido con la llegada de las tecnologías digitales han cambiado profundamente la forma en que las personas viven se relaciona y aprenden. Esto generó un nuevo orden social: la Cultura Digital, que se presenta como una nueva conformación social en la época contemporánea y que Escuela y Educadores no deben ignorar, ya que sus actores más expresivos, los jóvenes, son los más inmersos en ella.

Aún hoy, los educadores nos enfrentamos a la "aparente" disputa desigual de que luchamos por la atención de nuestros estudiantes, especialmente los adolescentes, ante el irresistible atractivo de los recursos sonoros e imágenes que permiten los gadgets. El desafío actual que se nos impone es replantear nuestra práctica pedagógica para estar al día con los grandes cambios que las tecnologías digitales han provocado en la sociedad actual.

La escuela actual aún conserva algunas prácticas pedagógicas basadas en el modelo educativo utilizado en el siglo XVIII con planes de estudio cerrados, organizados por materias y contenidos específicos. En esta perspectiva, el uso de tecnologías en el contexto escolar aún mantiene la lógica de distribución de la información sin una preocupación sistemática por hacerlas significativas. De esta manera, la escuela se aleja de las transformaciones sociales y culturales que se están produciendo en la sociedad contemporánea, creando así una brecha entre el mundo de la escuela y el mundo de la vida. Por tanto, es necesario repensar el modelo comunicacional del aula, para una sala en la que exista la coautoría entre docentes y alumnos, interacción entre las partes para la construcción del aprendizaje.

Cambiar las formas de comunicación y producción de conocimiento es fundamental para la educación de niños y jóvenes que ya están inmersos en la comunicación digital e interactiva fuera del ámbito escolar. Es en vista de este escenario y estos hallazgos que abogamos por la importancia de las prácticas gamificadas en la praxis pedagógica. El discurso esbozado hasta ahora sobre las tecnologías digitales se debe a que, entre todas ellas, los juegos son los que más seducen y ocupan espacio y tiempo en la vida de nuestros estudiantes. Asimismo, queremos dejar claro que el esfuerzo que se hace en este texto por caracterizar la importancia de los juegos en entornos virtuales no pretende proponer la transformación de la escuela en un juego. Pero primero, se pretende demostrar las posibilidades de cambios que la inserción de prácticas gamificadas puede representar para el aprendizaje escolar.

Sin embargo, para que esto suceda, los profesores y educadores deben comprender su concepción y lógica de funcionamiento. Y, hasta donde se sabe, la única forma posible de que esto suceda es conocer la dinámica de los juegos en entornos virtuales. Debe quedar claro que una actividad ludificada no es simplemente una actividad animada, lúdica. La gamificación presupone la presencia de elementos de juego, con una estructura narrativa con principio, medio y final, para mantener al jugador dentro del círculo mágico y motivado para aprender más y más.

\section{REFERENCIAS}

Álvarez, H. A. (2020). Enseñanza de la historia en el siglo XXI: Propuestas para promover el pensamiento histórico. Revista de Ciencias Sociales, XXVI(2), 442-459. doi:https://doi.org/10.31876/rcs.v26i0.34138

Alves, F. (2015). Gamification: Como criar experiências de aprendizagem engajadoras. DVS Editora. Obtenido de https://bit.ly/3sIQeEd

Burke, B. (2015). Gamificar: como a gamificação motiva as pessoas a fazer coisas extraordinárias. São Paulo: Editora DVS.

Carrión, E. (2018). El uso de la Gamificación y los recursos digitales en el aprendizaje de las Ciencias Sociales en la Educación Superior. DIM: Didáctica, Innovación y Multimedia, 15(36), 1-14.

Contreras, R. S., \& Eguia, J. L. (2017). Experiencias de gamificación en las aulas. Bellaterra: Institut de la Comunicació.: InCom-UAB Publicacions, 15. 
Contreras-Espinosa, R. (2016). Juegos digitales y gamificación aplicados en el ámbito de la educación. RIED. Revista Iberoamericana de Educación a Distancia, 19 (2), 27-33.

Cuenca, J. M., \& Jiménez, R. (2018). Enseñando historia y patrimonio a través de los videojuegos: investigación e innovación. Rivista dell'Istituto di Storia dell'Europa Mediterranea, 2(II), 4364. doi:https://doi.org/10.7410/1353

Deterding, S. (2012). Gamification: designing for motivation. Interactions, New York, v. 19, 14-17.

Dichev, C., \& Dicheva, D. (2017). Gamifying education: what is known, what is believed and what remains uncertain: a critical review. doi:10.1186/s41239-017-0042-5

Fardo, M. (2013). La gamificación como método: Estudio de elementos de juegos aplicados en procesos de enseñanza y aprendizaje. Rio Grande del Sur: Universidad de Caxias. Obtenido de https://bit.ly/3rbya5p

García, A. (27 de octubre de 2019). Videojuegos: una industria de 135.000 millones. Obtenido de http://www.magazinedigital.com/historias/reportajes/videojuegos-una-industria-135000millones

Gatti, L., Ulrich, M., \& Seele, P. (2019). Education for sustainable development through business simulation games: An exploratory study of sustainability gamification and its effects on students' learning outcomes. Journal of cleaner production, 207, 667-678. doi:https://doi.org/10.1016/j.jclepro.2018.09.130

Gee, J. P. (2009). Bons Videojogos + Boa Aprendizagem. Edições Pedago.

Harvey, D. (1990). The Condition of Postmodernity. An Enquiry into the Origins of Cultural Change. Oxford, Inglaterra: Amorrortu editores. Obtenido de https://bit.ly/3baWRcG

Huizinga, J. (2010). Homo ludens. Alianza Editorial. Obtenido de https://bit.ly/3e 3e9Kj

Iquise, M. E., \& Rivera, L. G. (2020). La importancia de la gamificación en el proceso de la enseñanza y aprendizaje. Universidad San Ignacio de Loyola.

Kapp, K. M. (2012). The gamification of learning and instruction : game-based methods and strategies for training and education. San Francisco, CA: Pfeiffer.

Kim, J. T., \& Lee, W. H. (2015). Dynamical model for gamification of learning (DMGL). Multimedia Tools and Applications, 74(19), 8483-8493. doi:https://doi.org/10.1007/s11042-013-1612-8

Martínez, G. (2017). Tecnologías y nuevas tendencias en educación: Aprender jugando. El caso de Kahoot. Opcion, 33(83), 252-277.

Martins, T. (2013). A metamorfose do modo de ser e de estar no mundo atual e as reais mudanças na sala de aula presencial. Revista Educação On-line PUC-Rio $n^{o} 12$, 150-166. Obtenido de http://www.maxwell.lambda.ele.pucrio.br/rev_edu_online.php?strSecao=input0.

Massarolo, J. C., \& Mesquita, D. (2013). Narrativa transmídia e a Educação: panorama e perspectivas. Revista Ensino Superior Unicamp. Obtenido de https://www.revistaensinosuperior.gr.unicamp.br/edicoes/edicoes/ed09_abril2013/NMES_3. pdf

McGonigal, J. (2012). Realidade em jogo: por que os games nos tornam melhores e como eles podem mudar o mundo. Rio de Janeiro: Best Seller.

Meşe, C., \& Dursun, Ö. Ö. (2018). Influence of Gamification Elements on Emotion, Interest and Online Participation. Egitim ve Bilim, 43(196). doi:10.15390/EB.2018.7726

Mite, M. A. (2020). Percepción de los docentes hacia la incorporación de estrategias de gamificación y videojuegos. Universidad Casa Grande.

Oliva, H. A. (2016). La gamificación como estrategia metodológica en el contexto educativo universitario. Realidad y reflexión, 44, 29-47.

Ortiz, A. M., Jordán, J., \& Agredal, M. (2018). Gamificación en educación: una panorámica sobre el estado de la cuestión. Educação e Pesquisa, 44(0), 1-17. doi:https://doi.org/10.1590/s16784634201844173773

Prensky, M. (2006). “Don't bother me mom - I'm learning!’. St. Paul: Paragon House. Obtenido de https://bit.ly/3e235x7 


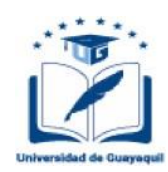

Putz, L. M., Hofbauer, F., \& Treiblmaier, H. (2020). Can gamification help to improve education? Findings from a longitudinal study. Computers in Human Behavior, 110, 106392. doi:https://doi.org/10.1016/j.chb.2020.106392

Rodríguez, F., \& Santiago, R. (2015). Gamificación. Cómo motivar a tu alumnado y mejorar el clima en el aula. Barcelona (España): OCEANO S.L.U.

Rodríguez, F., \& Santiago, R. (2015). Gamificación: Como motivar a tu alumnado y mejorar el clima en el aula. Editorial Océano.

Sánchez, Á., \& Colomer, J. C. (2018). Gamificación y construcción del pensamiento histórico: desarrollo de competencias en actividades gamificadas. CLIO. History and History teaching, 44, 82-93.

Sánchez-Pacheco, C. (2020). Los orígenes y el futuro de la gamificación. Guayaquil: Editorial Académica Española.

Sánchez-Pacheco, C. L. (2019). Gamificación: Un nuevo enfoque para la educación ecuatoriana. Revista Internacional Docentes 2.0 Vol. 7 Núm. 2, 96-105. Obtenido de https://bit.ly/3q830KU

Simões, J., Redondo, R. D., \& Fernández Vilas, A. (2013). A social gamification framework for a K6 learning platform. Computers in Human Behavior Volume 29, Issue 2, 345-353. doi:https://doi.org/10.1016/j.chb.2012.06.007

Vera, J. N. (2020). Gaming: una aproximación crítica desde la narrativa social contemporánea del nuevo orden mundial. Dictamen Libre, 27, 221-245. doi:https://doi.org/10.18041/26194244/d1.27.6649

Werbach, K., \& Hunter, D. (2012). For the win: how game thinking can revolutionize your business. Philadelphia: Wharton Digital Press.

Yee, N. (2007). Motivations for Play in Online Games. CyberPsychology \& Behavior Volume 9, Number 6, 772-775.

Zepeda, S., Abascal, R., \& López, E. (2016). INTEGRACIÓN DE GAMIFICACIÓN Y APRENDIZAJE ACTIVO EN EL AULA. Ra Ximhai, vol. 12, núm. 6, julio-diciembre, 2016, 315-325.

Zichermann, G., \& Cunningham, C. (2011). Gamification by Design. Canada: O'Reilly Media. Obtenido de https://www.pdfdrive.com/gamification-by-design-e39666432.html

Zúñiga, S. (2019). Percepción de los docentes hacia la incorporación de estrategias de gamificación y videojuegos. Universidad Casa Grande. 


\section{ANEXOS}

Tabla 1.

Ejemplos de elementos del juego.

Elementos

Narrativa

Niveles

Desafío / Misiones

Normas

Realimentación

Competencia

Compromiso

Recompensa

Puntuación / progresión
Descripción

Historia que promueve la inmersión del jugador en el juego.

División del juego en partes, generalmente con dificultades incrementales; también llamadas fases.

Objetivos que debe alcanzar el jugador.

Restricciones o limitaciones impuestas por el juego.

Respuesta a la acción de un jugador, que permite de inmediato la confirmación o reevaluación de opciones y tácticas.

Relación entre jugadores o equipos, que promueve la búsqueda de ser los mejores. Si está bien estimulado, puede promover innumerables aprendizajes. También se puede competir con uno mismo en la búsqueda de la superación.

Qué motiva al jugador a jugar.

Beneficio adquirido después de alguna acción o finalización de una misión.

Forma cuantificable del estado del juego.

Fuente: (Fardo, 2013)

CONFLICTOS DE INTERESES

Los autores no refieren conflictos de intereses 